\title{
Polarimetric Interferometric Synthetic Aperture Microscopy: Vectorial Computed Imaging from Optical Coherence Tomography Data
}

\author{
Brynmor J. Davis, Tyler S. Ralston, Daniel L. Marks, Stephen A. Boppart and P. Scott Carney \\ The Beckman Institute for Advanced Science and Technology and the Department of Electrical and Computer Engineering \\ The University of Illinois at Urbana-Champaign, 405 North Mathews Avenue, Urbana IL 61801 \\ bryn@uiuc.edu
}

\begin{abstract}
Interferometric Synthetic Aperture Microscopy (ISAM) obviates the trade-off between depth-of-focus and resolution in interferometric coherence imaging. In this work, ISAM's quantitative image reconstruction techniques are applied in a vectorial setting, thus admitting polarization-sensitive imaging.

(C)2007 Optical Society of America

OCIS codes: (100.3190) Inverse Problems; (100.6890) Three-dimensional Image Processing; (170.1650) Coherence Imaging; (170.4500) Optical Coherence Tomography; (110.6880) Three-dimensional Image Acquisition; (180.3170) Interference Microscopy; (120.5410) Polarimetry.
\end{abstract}

\section{Introduction}

Optical coherence tomography (OCT) [1] is a non-invasive technique that allows three-dimensional in-vivo imaging of tissue. Following the accepted description of focused light, the depth-of-focus in OCT has traditionally been regarded as fundamentally linked to the en-face resolution. Using a high numerical aperture (NA) lens provides a higher resolution, but at the expense of the image's depth-of-field. Like other lens-based three-dimensional imaging systems, such as confocal microscopy [2], the use of a high-NA lens in OCT [3] results in an image that is considered to be restricted to a single en-face plane. Alternatively the focal plane may be scanned to construct a three-dimensional image but at the expense of imaging speed.

A new computed imaging technique, interferometric synthetic aperture microscopy (ISAM) [4,5], has been used to overcome the trade-off between resolution and depth-of-focus. This technique implements a computational focusing on interferometric broadband data collected with a single focal plane - data such as that collected in OCT. ISAM includes a reference structure above the object in order to collect a phase-stable data set. Standard processing of the interferometric data results in a complex signal directly related to the scattered field [6]. Access to both amplitude and phase information allows the quantitative reconstruction of an image that has a resolution independent of the distance from the focal plane. The ISAM reconstruction approach has the advantage of being implemented using only simple scaling and resampling operations in the Fourier domain.

The derivation of ISAM reconstruction algorithms has recently been extended to include the vectorial nature of the electromagnetic fields used and arbitrary focusing apertures [7]. As the polarization state of the light is now explicitly included in the model, there is the potential for exploiting anisotropic scattering and propagation as contrast mechanisms - i.e. polarimetry can be performed. Polarimetry has been successfully employed in a number of fields using a variety of techniques (see [8] for a recent implementation), including in biomedical imaging using polarization-sensitive OCT [9]. This work presents initial results in the investigation of polarization-sensitive ISAM for quantitative polarimetric image reconstruction without depth-of-focus/resolution limitations.

\section{Anisotropic Scattering in Interferometric Synthetic Aperture Microscopy}

The object measured in ISAM can be described by a scattering potential $\eta$ that varies spatially. In the vectorial model presented in [7], $\eta$ is a tensor function of position, so that the effects of anisotropic scattering on polarization state can be modeled. The observation model was shown to be well approximated by,

$$
\tilde{S}\left(\mathbf{Q}_{\|}, k, \mathbf{E}^{i}, \mathbf{E}^{d}\right)=H_{\alpha \beta}\left(-\mathbf{Q}_{\|}, k, \mathbf{E}^{i}, \mathbf{E}^{d}\right) \tilde{\tilde{\eta}}_{\alpha \beta}\left(\mathbf{Q}_{\|},-2 k_{z}\left(\mathbf{Q}_{\|} / 2\right)\right),
$$

where a single tilde represents a Fourier transform with respect to the en-face dimensions, two tildes represent a three-dimensional spatial Fourier transform, $k$ is the measured wavenumber, $\mathbf{Q}_{\|}$are the en-face Fourier axes, $\mathbf{E}^{i}$ and $\mathbf{E}^{d}$ are the illumination and detection field directions, $k_{z}=\left(k^{2}-\left|\boldsymbol{Q}_{\|}\right|^{2}\right)^{0.5}, S$ is the complex data collected, $H$ is the instrument transfer function and $\alpha, \beta$ index the tensor components of the scattering potential. Note that Einstein 

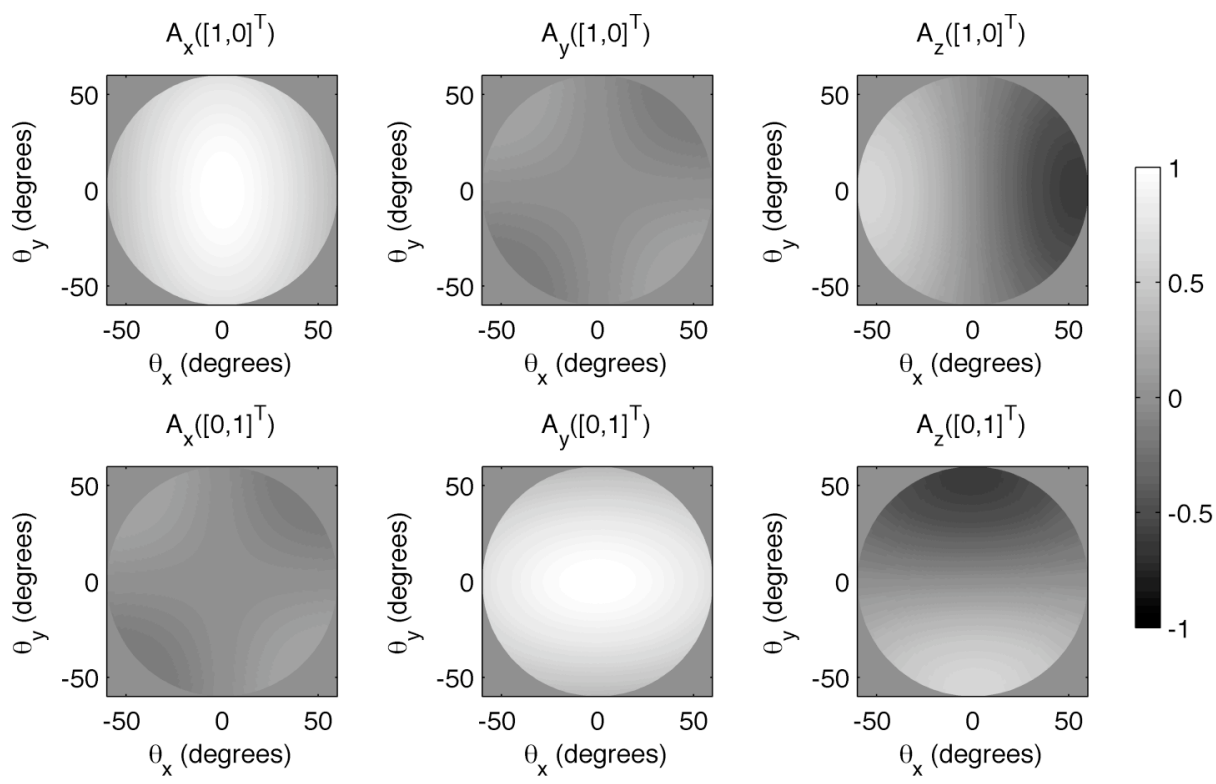

Fig. 1. Aperture fields as a function of the angle to focus and the incident field direction. An aplanatic lens with a numerical aperture of 0.87 is shown and the incident fields are x-polarized (top row) and y-polarized (bottom row).

summation is employed in Eq. (1). A linear susceptibility is assumed, as is the validity of the first Born approximation.

ISAM is an image reconstruction technique where data typically regarded as out-of-focus is computationally focused using shift-varying processing. The shift-varying part of the ISAM algorithm is characterized in the Fourier domain as,

$$
\begin{aligned}
k \rightarrow & -2 k_{z}\left(\mathbf{Q}_{\|} / 2\right), \\
= & -2 \sqrt{k^{2}-Q_{x}^{2} / 4-Q_{y}^{2} / 4} .
\end{aligned}
$$

As can be seen from Eq. (1), this places the data and the scattering potential on the same coordinate axes. The Fourier resampling produces a dramatic focusing of the data that would appear out of focus in OCT.

In scalar ISAM, the transfer function $H$ is inverted using linear, shift-invariant filtering. In the vectorial case, $H$ takes on a more significant role as it describes the relation between the illumination and detection polarization states $\left(\mathbf{E}^{i}\right.$ and $\left.\mathbf{E}^{d}\right)$ as well as the components of the tensor scattering potential $\eta$. As with related analysis [10], the values of the vectorial optical transfer function depend directly on the field at the exit pupil of the objective lens. This field will be denoted by $A_{\alpha}\left(\sin \left(\theta_{x}\right), \sin \left(\theta_{y}\right), \mathbf{E}, k\right)$, where $\theta_{x}$ and $\theta_{y}$ are the angles to the focal point, $\mathbf{E}$ is the incident field and $\alpha$ indexes the field direction on the exit pupil. It will be assumed that the lens is infinity-corrected, so the incident field $\mathbf{E}$ impinges on a planar entrance pupil and is therefore restricted to the en-face $(x, y)$ directions. The mathematics describing $A$ can be found in [11] and example calculations for an aplanatic lens are shown in Fig. 1.

The relation between the transfer function $H$ and the field in the aperture $A$ is given in [7] and is shown to be dependent on whether the scatterer in consideration is near the focus or far from it. In the near-focus case,

$$
H_{\alpha \beta}^{N}\left(\mathbf{Q}_{\|}, k, \mathbf{E}^{i}, \mathbf{E}^{d}\right) \approx \int A_{\alpha}\left(\frac{\mathbf{q}_{\|}}{k}, \mathbf{E}^{d}\right) A_{\beta}\left(\frac{\mathbf{Q}_{\|}-\mathbf{q}_{\|}}{k}, \mathbf{E}^{i}\right) d^{2} q_{\|} .
$$

This expression is calculated assuming that the lens aperture is rotationally symmetric. Equation (3) derived by taking the first term in a series expansion of $H^{N}$ that is given by the moments of the integrand above. In the far-fromfocus case,

$$
H_{\alpha \beta}^{F}\left(\mathbf{Q}_{\|}, k, \mathbf{E}^{i}, \mathbf{E}^{d}\right) \approx A_{\alpha}\left(\frac{\mathbf{Q}_{\|}}{2 k}, \mathbf{E}^{d}\right) A_{\beta}\left(\frac{\mathbf{Q}_{\|}}{2 k}, \mathbf{E}^{i}\right) .
$$


Constants of proportionality have been omitted from both Eq. (3) and Eq. (4), while a linear decay with $z$, the distance from the focus, is omitted in Eq. (4). The dependence upon a single $\mathbf{Q}_{\|}$value seen in Eq. (4) can be interpreted from a geometrical optics standpoint and is derived using the stationary phase approximation.

A quantitative relationship between the tensor scattering properties of the sample and the collected data are given by Eq. (1), Eq. (3) and Eq. (4). This gives a rigorous framework for ISAM polarimetry - that is, using multiple values of $\mathbf{E}^{i}$ and $\mathbf{E}^{d}$ to probe the full tensor nature of the object $\eta$. The effects of using partially-polarized or unpolarized probing light can also be analyzed using this framework and the appropriate statistical procedures [12]. It can be seen in Fig. 1 that with the use of a high-NA lens it is possible to produce significant fields in each of the $x$, $y$ and $z$ directions, while symmetries in the susceptibility [13] can be exploited to restrict the total number of unknown parameters in $\eta$. This leads to the expectation that significant polarimetric information can be gathered about the object.

However, it should also be noted that there will be unobservable quantities within the object. For example, it is not possible to produce a $z$-oriented field at the center of a standard lens, as at this point the incident ray is traveling in the $z$ direction. Equation (4) then dictates that $H$ at $\mathbf{Q}_{\|}=\mathbf{0}$ cannot be non-zero if either $\alpha$ or $\beta$ is equal to $z-$ this indicates an unobservable component of the object. While the formulation of polarimetric ISAM is well defined, this is an indication of how the achievable imaging performance has yet to be fully quantified.

\section{Conclusions and Outlook}

Interferometric synthetic aperture microscopy allows a quantitative, high-resolution, three-dimensional image to be constructed from interferometric broadband data collected with a single en-face scan. This work shows that this attractive functionality can be extended to polarimetric imaging. While polarimetric ISAM imaging has yet to be fully explored, initial results appear promising due to the ability to produce a wide range of polarization states within the object and measure the resulting backscattered light in a polarization-sensitive manner. Additional future work may also include the characterization of ISAM imaging in objects with anisotropic backgrounds (e.g. birefringent matertials) or the development of dedicated lens apertures [14].

\section{References}

[1] D. Huang, E. A. Swanson, C. P. Lin, J. S. Schuman, W. G. Stinson, W. Chang, M. R. Hee, T. Flotte, K. Gregory, C. A. Puliafito and J. G. Fujimoto, "Optical coherence tomography," Science 245, 1178-1181 (1991).

[2] T. Wilson and C. J. R. Sheppard, Theory and Practice of Scanning Optical Microscopy (Academic Press, 1984).

[3] J. A. Izatt, M. R. Hee,, G. M. Owen, E. A. Swanson and J. G. Fujimoto, "Optical coherence microscopy in scattering media," Opt. Lett. 19, 590-592 (1994).

[4] T. S. Ralston, D. L. Marks, P. S. Carney, S. A. Boppart, "Inverse scattering for optical coherence tomography," J. Opt. Soc. Am. A 23, 10271037 (2006).

[5] T. S. Ralston, D. L. Marks, P. S. Carney and S. A. Boppart, "Interferometric Synthetic Aperture Microscopy,” Nat. Phys. 3, 129-134 (2007).

[6] P. Hariharan, Optical Interferometry (Academic Press, 2003).

[7] B. J. Davis, S. C. Schlachter, D. L. Marks, T. S. Ralston, S. A. Boppart and P. S. Carney, "Non-paraxial vector-field modeling of optical coherence tomography and interferometric synthetic aperture microscopy," J. Opt. Soc. Am A (submitted), (2006).

[8] M. Mujat, E. Baleine and A. Dogariu, "Interferometric imaging polarimeter," J. Opt. Soc. Am. A 21, 2244-2249 (2004).

[9] J. F. de Boer and T. E. Miller, "Review of polarization sensitive optical coherence tomography and Stokes vector determination," J. Biomed. Opt. 7, 359-371 (2002).

[10] M. R. Arnison and C. J. R. Sheppard, "A 3D vectorial optical transfer function suitable for arbitrary pupil functions," Opt. Commun. 211, 53-63 (2002).

[11] B. Richards and E. Wolf, "Electromagnetic diffraction in optical systems. II. Structure of the image field in an aplanatic system," Proc. R. Soc. London A 253, 358-379 (1959).

[12] C. Brosseau, Fundamentals of Polarized Light: A Statistical Optics Approach (Wiley 1998).

[13] Y. R. Shen, The Principles of Nonlinear Optics (Wiley 2003).

[14] A. F. Abouraddy and K. C. Toussaint, Jr., “Three-dimensional polarization control in microscopy,” Phys. Rev. Lett. 96 (153901), (2006). 